\title{
An Omnidirectional Electromagnet for Remote Manipulation
}

\author{
Andrew J. Petruska and Jake J. Abbott
}

\begin{abstract}
An Omnimagnet is an omnidirectional electromagnet comprised of a ferromagnetic core inside of three orthogonal nested solenoids. It generates a magnetic dipole-field with both a variable dipole-moment magnitude and orientation with no moving parts. The design of an Omnimagnet, in which each solenoid has the same dipole moment and minimizes the differences between each of the solenoid's fields, is provided and optimized for strength by tailoring the size of the spherical core used to amplify the solenoids' field. This design is then analyzed using FEA tools and shown to be dipole-like in nature. Various magnetic control methods are then motivated by providing the necessary equations relating the three applied currents to applied field, torque, or force on an adjacent magnetic device. Finally, the optimal design is constructed and its utility is demonstrated by driving a helical capsule endoscope mockup through a transparent lumen.
\end{abstract}

\section{INTRODUCTION}

Magnetic microscale and mesoscale devices (both tethered and untethered) can be manipulated with an externally generated magnetic field, which applies a combination of force and torque to the device without any mechanical connection. Although a combination of permanent magnets and electromagnets can be used to produce the magnetic field required for a manipulation task, some tasks seem better suited to either permanent magnet or electromagnet systems. Because they have more direct real-time control of the applied magnetic field, electromagnet systems have been used for multidegree-of-freedom levitation and position/orientation control [1]-[4]. Permanent magnets, which require no electrical power to generate a strong field, have been used for pulling and rolling tasks in which the environment provides some structure [5]-[8], as well as for quasistatic pointing tasks of tethered devices such as magnetic catheters [9]. Because both attractive and lateral forces can be generated between a rotating dipole source and a sympathetically rotating magnetic device, a rotating dipole field could be more effective for rolling/screwing propulsion than the rotating uniform field generated by many electromagnet systems [10]. Finally, it is challenging to scale many laboratory electromagnetic systems that surround their workspace (e.g., Helmholtz coils) to a size that would be required for medical applications, whereas manipulation systems that utilize dipole fields can be located adjacent to their workspace.

An omnidirectional electromagnet, formed by any set of collocated electromagnets that have dipole moments spanning $\mathbb{R}^{3}$, combines the real-time control of field strength

This material is based upon work supported by the National Science Foundation under Grant Nos. 0952718 and 0654414

The authors are with the Department of Mechanical Engineering, University of Utah, Salt Lake City, 84112. \{andrew.petruska, jake.abbott\} @utah. edu

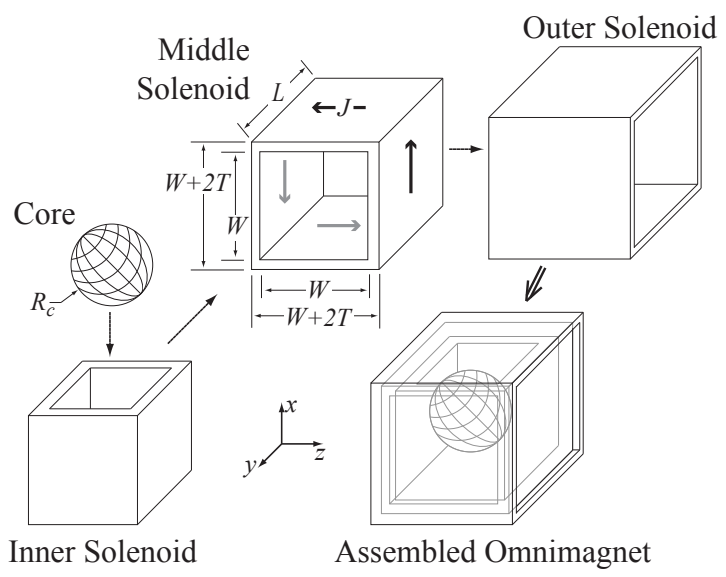

Fig. 1. The assembled Omnimagnet forms a cube constructed of three nested orthogonal solenoids surrounding a spherical core of ferromagnetic material. Each square-cross-section solenoid has a different inner width $W$, winding thickness $T$, length $L$, and associated current density $J$.

associated with traditional electromagnets and the control of dipole orientation associated with rotating permanent magnets. In this paper, we introduce a class of omnidirectional electromagnet that we call an Omnimagnet, comprised of a ferromagnetic core surrounded by three orthogonal solenoids, which is able to create a dipole-like field in any orientation. Specifically, we consider an Omnimagnet comprised of a spherical core and square-cross-section solenoids (Fig. 1), but other design variations could be considered. An Omnimagnet contains no moving parts, and when powered down becomes inert, reducing the safety concerns associated with permanent-magnet field sources. The concept of three nested solenoids has been explored as a method of inductive power coupling [11], but never as a dipole-like magneticfield source, and never with a spherical core.

The paper is structured as follows. First, the general design problem for an Omnimagnet is presented. Next, the magnetic fields generated by the three solenoids are described using a multipole expansion of the magnetostatic equations, and the contribution of the ferromagnetic core is quantified. The optimization of a specific Omnimagnet follows, and the design is described. The field generated by this design is then compared to a dipole-field approximation, and the inverse solution for determining the dipole moment, and thus the currents, required to produce a desired static or rotating field, torque, or force at a given location are provided. Finally, the capability of the Omnimagnet for the control of capsule endoscopes is then demonstrated, and future research plans are discussed. 


\section{OMnimagnet Design AND OPTIMIZATION}

The general concept of an Omnimagnet is broad, consisting of three orthogonal nested solenoids surrounding a ferromagnetic core; however, design choices must be made to realize and optimize a physical Omnimagnet. First, we chose the shape of the solenoids to be square-cross-sectional sleeves to result in a dense packing (see Fig. 1). Next, we chose the core to be a sphere because a spherical core has three desirable properties:

- A sphere does not have a preferential magnetization direction.

- When placed in a uniform field (similar to the field in the center of a solenoid), a sphere produces a pure point-dipole field [12].

- The average applied magnetic field within a sphere is equal to the applied magnetic field at the center of the sphere [12].

We chose that the dipole moment generated in each direction, which consists of the contribution of both an individual solenoid and the magnetization of the core due to that solenoid, should be the same when an equal electrical current density is applied through each solenoid. Other geometric design choices (e.g., cylindrical solenoids or a cubic core) or dipole-moment relationships (e.g., scaling the dipole moment of each solenoid with its heat-transfer capability), could also be pursued using the general framework for Omnimagnet design outlined below. Finally, we constrain our design to use a single wire gauge for all solenoids, which means that "the same current density" is synonymous with "the same current"; current and current density are related by the crosssectional area of the wire used. Throughout this paper, $I$ will be used to refer to currents in units $\{\mathrm{A}\}$ and $J$ will be used to refer to current density in units $\left\{\mathrm{A} \cdot \mathrm{m}^{-2}\right\}$. Because current density is invariant to wire selection, the optimization for shape is performed using $J$; general discussion, however, will use $I$, as it is the more natural parameter from a control perspective. The final design of the Omnimagnet shown in Fig. 1 requires ten total constraints (the length, width, and thickness of each solenoid, and the radius of the core).

The magnetic field generated by the Omnimagnet can be represented by the field contributed by the magnetized spherical core superimposed with the field contributed by the solenoids. Modeling the total field can be performed using FEA tools with a resolution limited by the number of elements used. Alternatively, an analytical dipole approximation can be used to model the field. The dipole approximation provides a closed-form vector equation that can be used to calculate the field generated at a point, or inverted to determine the current necessary to create a particular field. The closer the Omnimagnet is to generating a pure dipole field, the better the algorithms based on this approximation will perform. By correctly choosing the solenoids' aspect ratios, the dipole-approximation error can be minimized as a part of the design optimization.

\section{A. Solenoid Multipole Field Expansion}

For positions outside of the Omnimagnet's minimumbounding sphere (i.e., the smallest sphere that the Omnimagnet can fit within), the solenoid fields can be represented by a multipole expansion of a vector potential [12]:

$$
\mathbf{B}(\mathbf{p})=\nabla \times \Psi(\mathbf{p})
$$

where

$$
\Psi(\mathbf{p})=\frac{\mu_{0}}{4 \pi} \sum_{n=0}^{\infty} \frac{1}{\|\mathbf{p}\|^{n+1}} \int_{\mathcal{V}_{s}} \mathbf{J}(\mathbf{r})\|\mathbf{r}\|^{n} P_{n}(\hat{\mathbf{p}} \cdot \hat{\mathbf{r}}) d V
$$

where $\mu_{0}=4 \pi \times 10^{-7} \mathrm{~T} \cdot \mathrm{m} \cdot \mathrm{A}^{-1}$ is the magnetic permeability of free space, $\mathbf{p}$ is the vector (with associated unit vector $\hat{\mathbf{p}}$ ) from the center of the Omnimagnet to the point of interest in units $\{\mathrm{m}\}, \mathbf{r}$ is the vector (with associated unit vector $\hat{\mathbf{r}}$ ) from the center of the Omnimagnet to the point in the solenoid being integrated, $\mathbf{J}(\mathbf{r})$ is the current density vector that points in the direction of the current flow, $\mathcal{V}_{s}$ represents the solenoid's volume, and $P_{n}()$ are the Legendre polynomials. Since the divergence of a magnetic field through a closed surface must be zero, all of the even terms (those corresponding to $P_{0}, P_{2}, \ldots$ ) must be zero, leaving only the odd terms. The first non-zero term in the multipole expansion (corresponding to $P_{1}$ ) is the dipole field, which can be expressed in a coordinate-free form as:

$$
\mathbf{B}(\mathbf{p})=\frac{\mu_{0}}{4 \pi\|\mathbf{p}\|^{3}}\left(3 \hat{\mathbf{p}} \hat{\mathbf{p}}^{\mathrm{T}}-\mathbb{I}\right) \mathbf{m}
$$

where $\mathbb{I}$ is a $3 \times 3$ identity matrix and $\mathbf{m}$ is the dipole moment in units $\left\{\mathrm{A} \cdot \mathrm{m}^{2}\right\}$.

The dipole moment for a current density of any configuration is [12]:

$$
\mathbf{m}=\frac{1}{2} \int_{\mathcal{V}_{s}} \mathbf{r} \times \mathbf{J}(\mathbf{r}) d V
$$

The dipole moment for a square-cross-section solenoid as shown in Fig. 1 with uniform current density (i.e., the current density does not vary along the thickness or length of the solenoid) is:

$$
\mathbf{m}=\frac{J L^{4}}{6}\left(\beta_{2}^{3}-\beta_{1}^{3}\right) \hat{\mathbf{l}}
$$

where $J$ is the magnitude of $\mathbf{J}, L$ is the axial length of the solenoid (with associated axial unit vector $\hat{\mathbf{l}}$ ), and $\beta_{1}=W / L$ and $\beta_{2}=(W+2 T) / L$ respectively describe the innerwidth-to-length and outer-width-to-length aspect ratios.

The maximum dipole moment that any electromagnet with a bounding cube of edge length $L$ containing no ferromagnetic material could generate in one direction can be calculated by (5) with $\beta_{1}=0, \beta_{2}=1$, and is $J L^{4} / 6$. The maximum theoretical dipole moment that could be expected for any cubic omnidirectional electromagnet with edge length $L$ containing no ferromagnetic material is thus $1 / 3$ of the unidirectional case: $J L^{4} / 18$; this quantity is used throughout the paper to normalize the strength for a nondimensional optimization, although constructing such an idealized omnidirectional electromagnet would be very challenging. 
We show in [13] that by varying the aspect ratios of a rectangular permanent magnet, the dipole-field approximation error can be minimized. The approach finds the geometry that sets the next term in the multipole expansion, the quadrupole term, to zero in the scalar potential of the magnetic field. Using the same technique, but using the vector potential instead of a scalar potential, the magnetic field of each solenoid can be shaped to produce a dipolelike field by removing the quadrupole contribution to the multipole expansion. The quadrupole term for a square-crosssection solenoid of uniform current density corresponds to the $P_{3}$ term in the expansion:

$$
\begin{aligned}
\mathbf{B}_{\text {quad }}(\mathbf{p})=\frac{\mu_{0}}{4 \pi} \frac{1}{\|\mathbf{p}\|^{5}} & \left(\left(35\left(\hat{\mathbf{m}}^{\mathrm{T}} \hat{\mathbf{p}}\right)^{2}-15\right) \hat{\mathbf{p}} \hat{\mathbf{p}}^{\mathrm{T}}\right. \\
- & \left.\left(15\left(\hat{\mathbf{m}}^{\mathrm{T}} \hat{\mathbf{p}}\right)^{2}-3\right) \mathbb{I}\right) \mathbf{m}_{\text {quad }}
\end{aligned}
$$

where $\mathbf{m}_{\text {quad }}$ is the quadrupole moment, given by:

$$
\mathbf{m}_{\text {quad }}=\frac{3}{40} L^{2}\left(\beta_{1}^{2}+\frac{\beta_{2}^{4}+\beta_{1} \beta_{2}^{3}}{\beta_{1}^{2}+\beta_{1} \beta_{2}+\beta_{2}^{2}}-\frac{5}{3}\right) \mathbf{m} .
$$

The values of $\beta_{1}$ and $\beta_{2}$ that set (7) to zero correspond to geometries with minimal dipole-field approximation error. By letting $\beta_{1}=\alpha \beta_{2}$ in (7) and equating to zero, it can be shown that the only meaningful solutions lie in the exclusive range $\beta_{2}=(1, \sqrt{5 / 3})$, which corresponds to geometries that are shorter than they are wide $(L<W+2 T)$. This requirement will be used to further constrain and simplify the optimization space.

\section{B. Core Dipole-Field Contribution}

Since the core is spherical and placed in the nearly uniform field inside of the solenoid, it is assumed that it will magnetize uniformly and contribute a pure dipole field (we verified this assumption numerically post facto and found it to be extremely accurate).

The dipole moment of a low-coercivity, low-remanence, and high-permeability $(\chi \gg 1)$ spherical core, when magnetized in its linear region, is

$$
\mathbf{m}_{c}=\overline{\mathbf{M}} \mathcal{V}_{c}=\left(\frac{\chi}{1+\frac{1}{3} \chi} \frac{\overline{\mathbf{B}}}{\mu_{0}}\right)\left(\frac{4 \pi}{3} R_{c}^{3}\right) \approx \frac{4 \pi R_{c}^{3}}{\mu_{0}} \mathbf{B}_{c}
$$

where $R_{c}$ is the radius of the core, $\mathrm{M}$ is the magnetization in units $\left\{\mathrm{A} \cdot \mathrm{m}^{-1}\right\}$, the overbar represents a quantity averaged over the core volume $\mathcal{V}_{c}$, and $\mathbf{B}_{c}$ is the applied magnetic field at the center of the core, which is a linear combination of the field due to each solenoid. The field, calculated by the Biot-Savart law, for each square-cross-section solenoid with uniform current density $J$, length $L$, and axis $\hat{\mathbf{l}}$ is:

$$
\mathbf{B}_{c}=\frac{2 L J \mu_{0}}{\pi} \int_{\beta_{1}}^{\beta_{2}} \operatorname{atan}\left(\frac{1}{\sqrt{1+2 \zeta^{2}}}\right) d \zeta \hat{\mathbf{l}}
$$

\section{Omnimagnet Optimization}

By combining the dipole moments due to the magnetized core and each of the solenoids, the total dipole moment of the Omnimagnet $\mathbf{m}=\mathbf{m}_{x}+\mathbf{m}_{y}+\mathbf{m}_{z}$ is thus:

$$
\begin{array}{r}
\mathbf{m}=\sum_{i \in\{x, y, z\}} J_{i}\left(8 L_{i} R_{c}^{3} \int_{\beta_{i, 1}}^{\beta_{i, 2}} \operatorname{atan}\left(\frac{1}{\sqrt{1+2 \zeta^{2}}}\right) d \zeta\right. \\
\left.+\frac{L_{i}^{4}}{6}\left(\beta_{i, 2}^{3}-\beta_{i, 1}^{3}\right)\right) \hat{\mathbf{l}}_{i}=\mathbb{M I}
\end{array}
$$

where the indices $x, y$, and $z$ correspond to the inner, middle, and outer solenoids, respectively and without loss of generality; and $\mathbb{M}$ is a linear transformation that maps the three applied currents in $\mathbf{I}$ to the dipole moment $\mathbf{m}$.

The optimal geometry for the Omnimagnet corresponds to the geometric ratios that maximize the dipole moment generated in each direction, have the same ratio of dipole moment to maximum current density in each direction, and have no quadruple moment. This is a constrained optimization problem, and can be non-dimensionalized by dividing all of the lengths by $L_{\max }$ (the edge length of a minimumbounding cube) and the moments by $m_{\text {ref }}=J_{\max } L_{\max }^{4} / 18$ (the maximum no-ferromagnetic-material dipole moment introduced earlier). The constraints can be simplified because we know from (7) that the length of each solenoid must be shorter than its outer width. Thus, the objective is to maximize the dipole moment magnitude $\|\mathbf{m}\|$ subject to:

- Eq. (7) equals zero (i.e., the configuration has no quadrupole moment).

- $\left\|\mathbf{m}_{x}\right\| / m_{\text {ref }}=\left\|\mathbf{m}_{y}\right\| / m_{\text {ref }}=\left\|\mathbf{m}_{z}\right\| / m_{\text {ref }}$.

- $W_{x}=R_{c}$ (i.e., the core diameter is the same as the inner solenoid's inner width).

- $W_{y}=W_{x}+2 T_{x}$ (the inner solenoid's outer width is the same size as the middle solenoid's inner width).

- $W_{z}=W_{y}+2 T_{y}$ (the middle solenoid's outer width is the same size as the outer solenoid's inner width).

This optimization is performed using $R_{c}$ as the free parameter (Fig. 2). There is a maximum that occurs when the core diameter is $60 \%$ of $L_{\max }$. Although the magnitude of the dipole moment in each direction is the same for the same applied current, the percentage of the dipole moment attributed to the core or the windings are different for each solenoid; the percentage of the dipole moment from the (core/windings) is (41/59), (28/72), and (21/79) for the inner, middle, and outer solenoids, respectively. Interestingly, this configuration has dipole-moment magnitudes that are 93\% of what could be theoretically expected with no ferromagnetic material and no voids (an unrealizable geometry), and $22 \%$ greater than the realizable geometry of three nested solenoids with no ferromagnetic core (but with significantly less power consumption and more heat-transfer surface area). Solutions to the right of the maximum in Fig. 2 correspond to geometries with more inert (non-current-energized) material and will produce less heat and require less power than the corresponding geometry left of the optimal point. The 


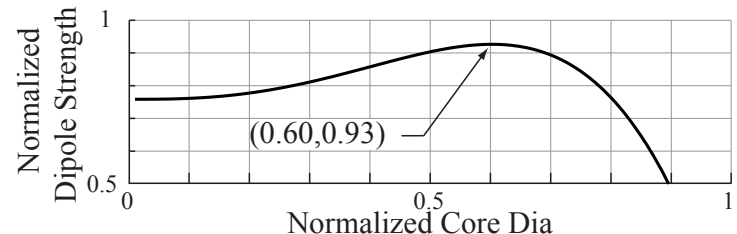

\begin{tabular}{|c|c|c|c|c|c|}
\hline & $L / L_{\max }$ & $W / L_{\max }$ & $T / L_{\max }$ & $\beta_{1}$ & $\beta_{2}$ \\
\hline $\mathrm{x}$ & 0.70 & 0.60 & 0.09 & 0.86 & 1.11 \\
\hline $\mathrm{y}$ & 0.84 & 0.78 & 0.06 & 0.92 & 1.07 \\
\hline $\mathrm{z}$ & 0.96 & 0.91 & 0.05 & 0.95 & 1.05 \\
\hline
\end{tabular}

Fig. 2. The optimal geometry for a no-quadrupole Omnimagnet. The dipole moments have been normalized by $J L_{\max }^{4} / 18$ and have a maximum at a core-diameter-to-outer-Omnimagnet-dimension ratio $2 R_{c} / L_{\max }=0.60$. The table provides the geometric ratios that describe the shape of the three nested solenoids that correspond to this optimal configuration. All length parameters are normalized by the outer Omnimagnet cubic length $L_{\max }$.

flatness of the maximum indicates that variations about the optimal point will not substantially affect the performance of the resulting Omnimagnet. Fortunately, the solenoids are shorter than they are wide, which allows paths for conductors and coolant to reach the middle and inner solenoids, making the implementation of this design feasible.

An Omnimagnet was constructed using a $100 \mathrm{~mm}$ diameter spherical Nickel-Iron (ASTM A753-08-K94840) core. The core material was chosen because it has a high magnetic permeability, a low magnetic remanence, and a low magnetic coercivity (i.e., it magnetizes easily but does not remain magnetized when the magnetizing field is removed). The solenoids were constructed using 16 AWG square selfbonding copper wire from MWS Wire Industries Inc. The optimization was repeated including a $1 \mathrm{~mm}$ spacing between each of the components for assembly. The slightly modified optimization did not change the shape of the coils substantially but shifted the optimal core size to $57 \%$ of the edge length of the minimum-bounding cube, which is $L_{\max }=$ $176 \mathrm{~mm}$. The additional empty space also reduced the overall strength of the design from $93 \%$ to $87 \%$ of $m_{\text {ref }}$. Because of the quantization in lengths and widths inherent with any winding, the constructed Omnimagnet has slight variations in the dipole-moment strengths of each solenoid and has successfully minimized, but not eliminated, the quadrupole term $\left(\mathbf{m}_{\text {quad }} \approx 0.04 \mathbf{m}\right)$. The geometry constructed is shown in Fig. 3, with the table providing the dimensions; the dipole-moment per conductor-current is calculated to be 25.1, 25.8, and $26.3\left(\mathrm{~A} \cdot \mathrm{m}^{2}\right) / \mathrm{A}$ for the inner, middle, and outer solenoids, respectively, which form the diagonal entries of $\mathbb{M}$. The field at the surface with $1 \mathrm{~A}$ applied is measured to be 5.6, 4.7, and $3.6 \mathrm{mT}$ for the inner, middle, and outer solenoids, respectively; at $12 \mathrm{~cm}$ from the surface the field is $0.6 \mathrm{mT}$ for each.

Since each solenoid in the Omnimagnet has a different geometry, the magnetic field produced by each solenoid will not have exactly the same shape for positions close to the Omnimagnet. To understand the subtle differences in field shape, FEA simulations were performed using Ansoft

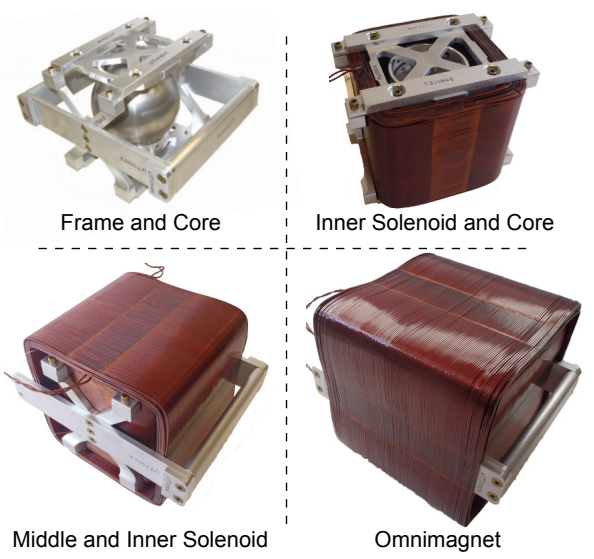

\begin{tabular}{|c|c|c|c|c|c|}
\hline & $L(\mathrm{~mm})$ & $W(\mathrm{~mm})$ & $T(\mathrm{~mm})$ & $\beta_{1}$ & $\beta_{2}$ \\
\hline $\mathrm{x}$ & 120 & 103 & 16 & 0.86 & 1.11 \\
\hline $\mathrm{y}$ & 149 & 137 & 11 & 0.92 & 1.07 \\
\hline $\mathrm{z}$ & 170 & 160 & 8 & 0.95 & 1.05 \\
\hline
\end{tabular}

Fig. 3. The assembled Omnimagnet used in the testing described in this paper. The core used is $100 \mathrm{~mm}$ which is $57 \%$ of $L_{\max }=176 \mathrm{~mm}$. The deviations from Fig. 2 are due to reoptimizing with $1 \mathrm{~mm}$ air-gaps between the components for assembly.
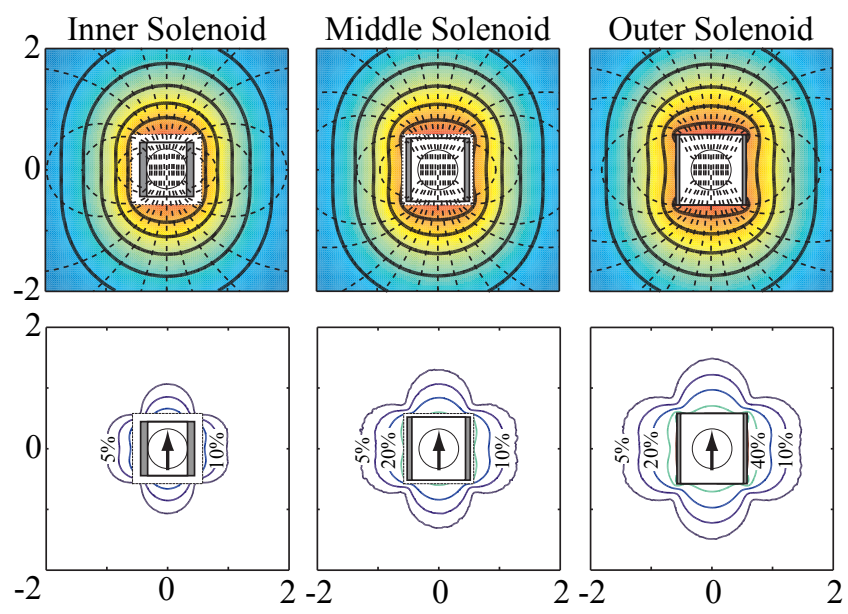

Fig. 4. The field shape (top, dotted) and magnitude (top, solid and color contour) and field error relative to the dipole approximation (bottom) are shown. The distances are normalized by the radius of a minimum-bounding sphere $\left(\sqrt{3} L_{\max } / 2\right)$. In the top row, the innermost contour line corresponds to $\|\mathbf{B}\|=64 \mu_{0}|\mathbf{m}|$, and each successively larger contour corresponds to a halving in field magnitude, with the outermost contour corresponding to $|\mathbf{B}|=4 \mu_{0}|\mathbf{m}|$.

Maxwell 14.0. Since the core is magnetized in the linear region and the solenoids are orthogonal, solenoid-solenoid magnetic coupling is negligible, so in these simulations only one of the solenoids is energized at a time. The results of the simulation (field strength, field shape, and percent error from the point-dipole approximation) for each solenoid are shown in Fig. 4. As the outermost solenoid is the largest, it is responsible for the majority of the field deviations close to the Omnimagnet. The field in each direction rapidly reduces to a pure dipole field with distance; the deviations are comparable to non-spherical permanent magnets [13]. 


\section{Manipulation With an OMnimagnet}

\section{A. Applying Field, Torque, or Force}

Fundamentally, the Omnimagnet is a magnetic field source with three control inputs (the current applied to each solenoid), which can be used to generate a desired magnetic field at a location in space, or control the torque or force on a magnetic object. Since the magnetic field generated is closely represented by a dipole field, at each location in space it will produce both a field and spatial derivatives in the field. With only a single Omnimagnet with three degrees of freedom, it is not possible to independently control field, torque, and force simultaneously. Moreover, it will be shown that it is not possible to fully control force in all configurations.

To determine the dipole moment $\mathbf{m}$ required by the Omnimagnet to produce a desired field $\mathbf{B}$ at some point $\mathbf{p}$, the vector dipole equation (3) must be inverted; Mahoney et al. showed the inverse always exists of the form [8]:

$$
\mathbf{m}=\frac{2 \pi}{\mu_{0}}\|\mathbf{p}\|^{3}\left(3 \hat{\mathbf{p}} \hat{\mathbf{p}}^{\mathrm{T}}-2 \mathbb{I}\right) \mathbf{B}
$$

Combining with (10), the currents required are thus:

$$
\mathbf{I}=\frac{2 \pi}{\mu_{0}}\|\mathbf{p}\|^{3} \mathbb{M}^{-1}\left(3 \hat{\mathbf{p}} \hat{\mathbf{p}}^{\mathrm{T}}-2 \mathbb{I}\right) \mathbf{B}
$$

If the tool's dipole moment $\mathbf{m}_{t}$ is known in addition to its position, it is possible to command torque or force directly, rather than simply setting a field value. The torque acting on the tool is

$$
\boldsymbol{\tau}=\mathbf{m}_{t} \times \mathbf{B}
$$

where $\times$ denotes a vector cross product. Although there is not a unique solution for the magnetic field to create a desired torque $\tau_{\text {des }}$, one simple method, which minimizes the field applied, is to command the field to be perpendicular to the tool:

$$
\mathbf{B}=\frac{1}{\left\|\mathbf{m}_{t}\right\|^{2}}\left(\boldsymbol{\tau}_{\mathrm{des}} \times \mathbf{m}_{t}\right)
$$

This method has also been shown to minimize the attractive force between the two dipoles [14].

To apply a controlled force for pushing and pulling tasks, the spatial gradient of the dipole-dipole potential energy must be controlled:

$$
\mathbf{F}=\frac{3 \mu_{0}}{4 \pi\|\mathbf{p}\|^{4}} \mathbb{F}\left(\mathbf{m}_{t}, \hat{\mathbf{p}}\right) \mathbf{m}
$$

and

$$
\mathbb{F}\left(\mathbf{m}_{t}, \hat{\mathbf{p}}\right)=\left[\begin{array}{c}
\mathbf{m}_{t}^{\mathrm{T}}\left(\hat{\mathbf{x}} \hat{\mathbf{p}}^{\mathrm{T}}+\hat{\mathbf{p}} \hat{\mathbf{x}}^{\mathrm{T}}-\left(5 \hat{\mathbf{p}} \hat{\mathbf{p}}^{\mathrm{T}}-\mathbb{I}\right) \hat{\mathbf{p}}^{\mathrm{T}} \hat{\mathbf{x}}\right) \\
\mathbf{m}_{t}^{\mathrm{T}}\left(\hat{\mathbf{y}} \hat{\mathbf{p}}^{\mathrm{T}}+\hat{\mathbf{p}} \hat{\mathbf{y}}^{\mathrm{T}}-\left(5 \hat{\mathbf{p}} \hat{\mathbf{p}}^{\mathrm{T}}-\mathbb{I}\right) \hat{\mathbf{p}}^{\mathrm{T}} \hat{\mathbf{y}}\right) \\
\mathbf{m}_{t}^{\mathrm{T}}\left(\hat{\mathbf{z}} \hat{\mathbf{p}}^{\mathrm{T}}+\hat{\mathbf{p}} \hat{\mathbf{z}}^{\mathrm{T}}-\left(5 \hat{\mathbf{p}} \hat{\mathbf{p}}^{\mathrm{T}}-\mathbb{I}\right) \hat{\mathbf{p}}^{\mathrm{T}} \hat{\mathbf{z}}\right)
\end{array}\right]
$$

where $\hat{\mathbf{x}}, \hat{\mathbf{y}}$, and $\hat{\mathbf{z}}$ are the cartesian $x, y$, and $z$ unit vectors, respectively. Since $\mathbb{F}$ is a $3 \times 3$ matrix, this inversion can be efficiently computed when the inverse exists. Since the orientation of the field, and therefore the torque applied, is not controlled, the resulting solution, when it exists, will likely be locally unstable. Consequently, force control with a single Omnimagnet is not feasible in practice.

\section{B. Propulsion of a Helical Capsule Endoscope Mockup}

At steady-state, the torque applied by a magnetic field on a magnetic tool will tend to align the tool with the field. This can be used to reduce the control problem from controlling torque directly, and thus requiring both tool orientation and position, to controlling field directly, and thus requiring only tool position. Controlling the propulsion of a ball or helical screw with this approach has been explored using a rotating permanent magnet [8]. However, when a permanent magnet is used to create a rotating field, both the magnitude and rotation rate of the field vary elliptically at the tool location; thus, the rotational speed of the permanent magnet needs to be constantly updated in order to produce a constant tool rotation rate [8]. Since the Omnimagnet can produce a desired field at a specific point in space, it is possible to create a rotating field with angular velocity $\boldsymbol{\omega}$ without the elliptical modulation associated with rotating permanent magnets. This can be accomplished by updating the desired B in (12) as

$$
\mathbf{B}(k+1)=e^{\mathbb{S}(\boldsymbol{\omega} \Delta t)} \mathbf{B}(k)
$$

where $\Delta t$ is the time step of the control system, $\mathbb{S}(\cdot)$ is the skew-symmetric matrix packing of a given angle-axis vector, and the matrix exponential creates a rotation matrix [15]. Thus, the same steady-state rotating control approachs can be performed by an Omnimagnet using (12) and (17) in which, unlike permanent magnets, both the desired field magnitude and orientation are specified. The minimum required field magnitude $\|\mathbf{B}\|$ is determined by (14) where $\left\|\tau_{\text {des }}\right\|$ is given by the viscous drag torque on the tool as a function of $\|\boldsymbol{\omega}\|$. In general, the larger the $\|\mathbf{B}\|$ chosen, the more robust the control will be to errors in modeling the viscous drag, but the greater the required Omnimagnet drive currents.

A rotating magnetic field was used to propel a threaded dummy capsule endoscope down a transparent lumen, which was offset by $12 \mathrm{~cm}$ from the surface of the Omnimagnet, as shown in Fig. 5. Although the trajectory of the capsule is simple (a line), the translating rotational field necessary to drive the capsule uses all three degrees of freedom available to the Omnimagnet (see multimedia attachment). The desired magnetic field with $\|\mathbf{B}\|=3 \mathrm{mT}$ was updated using (17) for a rotational rate of $2 \mathrm{~Hz}$. The position of the capsule was tracked using a stereo-vision system, although other localization methods, such as the magnetic localization of [16], could be used in the future. This position was used in conjunction with (12) to calculate the currents necessary to produce the desired field at the location of the capsule. The necessary currents were controlled by a DC voltage signal sent from a Sensory S626 controller card to Advanced Motion Control AMC16A8 current drives at an update rate of $100 \mathrm{~Hz}$.

\section{Discussion}

The Omnimagnet prototype developed in this paper uses no form of cooling. However, for Omnimagnets to be truly effective, they will need to be cooled. Future work will consider immersive fluid cooling and forced-convection fluid 


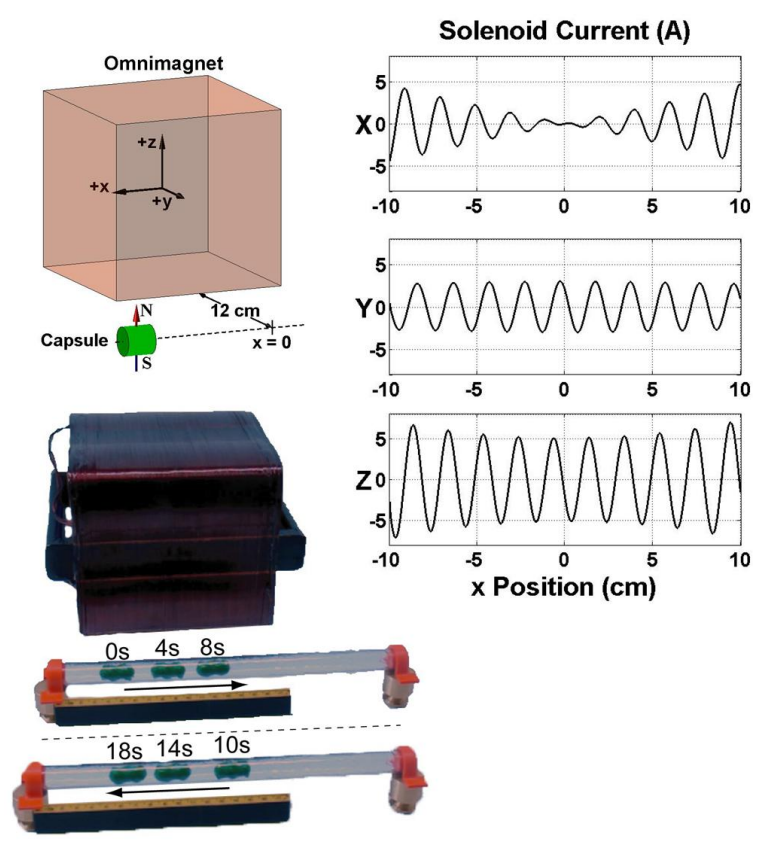

Fig. 5. Propulsion of a helical capsule at $1 \mathrm{~cm} \cdot \mathrm{s}^{-1}$ through a lumen located $12 \mathrm{~cm}$ from the surface of the Omnimagnet, which is applying a rotating magnetic field at the location of the helical capsule. (top) Numerical simulation of required solenoid currents. (bottom) Experimental demonstration.

cooling, which will enable higher currents, and therefore higher fields, to be generated. An Omnimagnet's dipole strength also increases with size, but this must be balanced against competing factors (e.g., size, weight., cost).

By combining multiple Omnimagnets together, it will be possible to create more sophisticated magnetic manipulation systems. For example, the Octomag system uses eight stationary electromagnets to generate 3-DOF force and 2-DOF torque on magnetic devices [3]. A system consisting of three Omnimagnets is essentially nine stationary electromagnets, meaning that a similar level of control as the Octomag seems conceivable. The commercial Stereotaxis Niobe system uses two large orientation-controlled permanent magnets to steer magnetic catheters. A system consisting of two Omnimagnets has the ability to recreate the same type of magnetic control. Additionally, the spherical cores of Omnimagnets makes their use in multi-Omnimagnet systems extremely promising; because the average magnetization of a spherical core can be solved using only knowledge of the applied field at the center of the sphere, it will be possible to solve for the combined field of multiple Omnimagnets analytically, rather than relying on in situ system calibration.

\section{CONClusions}

The design and optimization of an Omnimagnet was provided. The realized version of the design has an optimal core-radius to outer-length ratio of 0.6 and can achieve field strengths that are $87 \%$ of the unrealizable theoretical reference. The design was further optimized to create a dipole-like field with the error relative to the dipole-model falling to below $5 \%$ within 1.5 minimum-bounding-sphere radii from the center. Manipulation with the Omnimagnet was then demonstrated by actuating a helical capsule down a lumen. Not only can the Omnimagnet create a rotating dipole field like a permanent magnet, but it can also control the field strength like a standard electromagnet. This enables new control methodologies to be explored. Moreover, the spherical core will allow the combined field of multiple Omnimagnets to be solved analytically. Future work will include characterizing the differences between permanentmagnet actuation and Omnimagnet actuation of rotating tools, and exploring multiple-Omnimagnet manipulation.

\section{ACKNOWLEDGMENT}

The authors would like to thank Joseph Brink and Arthur Mahoney for their technical assistance.

\section{REFERENCES}

[1] S. Martel, C. C. Tremblay, S. Ngakeng, and G. Langlois, "Controlled manipulation and actuation of micro-objects with magnetotactic bacteria," Appl. Phys. Lett., vol. 89, no. 233904, 2006.

[2] S. Martel, J.-B. Mathieu, O. Felfoul, A. Chanu, E. Aboussouan, S. Tamaz, P. Pouponneau, L. Yahia, G. Beaudoin, G. Soulez, and M. Mankiewicz, "Automatic navigation of an untethered device in the artery of a living animal using a conventional clinical magnetic resonance imaging system," Appl. Phys. Lett., vol. 90, no. 114105 , 2007.

[3] M. P. Kummer, J. J. Abbott, B. E. Kratochvil, R. Borer, A. Sengul, and B. J. Nelson, "Octomag: An electromagnetic system for 5-dof wireless micromanipulation," IEEE Trans. Robot., vol. 26, no. 6, pp. 1006-1017, 2010.

[4] M. Mehrtash, N. Tsuda, and M. Khamesee, "Bilateral macro-micro teleoperation using magnetic levitation," IEEE-ASME Trans. Mech. vol. 16, no. 3, pp. 459-469, 2011.

[5] M. H. Hagiwara, T. K. Kawahara, Y. Yamanishi, and F. Arai, "Driving method of microtool by horizontally arranged permanent magnets for single cell manipulation," Appl. Phys. Lett., vol. 97, no. 013701, 2010.

[6] G. Ciuti, P. Valdastri, A. Menciassi, and P. Dario, "Robotic magnetic steering and locomotion of capsule endoscope for diagnostic and surgical endoluminal procedures," Robotica, vol. 28, pp. 199-207, 2010

[7] S. Yim and M. Sitti, "Design and rolling locomotion of a magnetically actuated soft capsule endoscope," IEEE Trans. Robot., vol. 28, no. 1, pp. 183-194, 2012.

[8] A. W. Mahoney, D. L. Cowan, K. M. Miller, and J. J. Abbott, "Control of untethered magnetically actuated tools using a rotating permanent magnet in any position," in IEEE Int. Conf. Robotics and Automation, 2012, pp. 3375-3380.

[9] M. N. Faddis, W. Blume, J. Finney, A. Hall, J. Rauch, J. Sell, K. T. Bae, M. Talcott, and B. Lindsay, "Novel, magnetically guided catheter for endocardial mapping and radiofrequency catheter ablation," Circulation, vol. 106, pp. 2980-2985, 2002.

[10] A. W. Mahoney and J. J. Abbott, "Managing magnetic force applied to a magnetic device by a rotating dipole field," Appl. Phys. Lett., vol. 99, no. 134103, 2011.

[11] R. Carta, J. Thoné, and R. Puers, "A wireless power supply system for robotic capsular endoscopes," Sens. Actuators, A, vol. 162, no. 2, pp. 177-183, 2010.

[12] D. J. Griffiths, Introduction to Electrodynamics. Prentice Hall, 1999.

[13] A. J. Petruska and J. J. Abbott, "Optimal permanent-magnet geometries for dipole field approximation," IEEE Trans. Magn., vol. 2, pp. 811-819, 2013.

[14] A. W. Mahoney, S. E. Wright, and J. J. Abbott, "Managing the attractive magnetic force between an untethered magnetically actuated tool and a rotating permanent magnet," in IEEE Int. Conf. Robotics and Automation, 2013.

[15] R. M. Murray, Z. Li, and S. S. Sastry, A Mathematical Introduction to Robotic Manipulation. CRC Press, Boca Raton, 1994.

[16] K. M. Miller, A. W. Mahoney, and J. J. Abbott, "Localization method for a magnetic capsule endoscope propelled by a rotating magnetic dipole field," in IEEE Int. Conf. Robotics and Automation, 2013. 